\title{
Implementing Appearance-Care Research for Breast Cancer Patient into Routine Clinical Practice
}

\author{
Mari Ikeda $^{1^{*}(D)}$, Hisako Kanai², Misato Osaka ${ }^{2}$, Nao Tamai ${ }^{3}$, Yukie Takemura ${ }^{4}$, Hiromi Sanada ${ }^{3}$ \\ ${ }^{1}$ Department of Nursing Administration, School of Nursing, Tokyo Women's Medical University, \\ Tokyo, Japan \\ ${ }^{2}$ Breast Center/Oncology Center, St. Luke’s International Hospital, Tokyo, Japan \\ ${ }^{3}$ Department of Gerontological Nursing/Wound Care Management, Graduate School of Medicine, The University of Tokyo, \\ Tokyo, Japan \\ ${ }^{4}$ Department of Nursing Administration, Graduate School of Medicine, The University of Tokyo, \\ Tokyo, Japan \\ Email: ^maritake-tky@umin.ac.jp, kanahisa@luke.ac.jp, miohat@luke.ac.jp, ntamai@m.u-tokyo.ac.jp, \\ yukitake-tky@umin.ac.jp, hsanada-tky@umin.ac.jp
}

How to cite this paper: Ikeda, M., Kanai, H., Osaka, M., Tamai, N., Takemura, Y. and Sanada, H. (2020) Implementing Appearance-Care Research for Breast Cancer Patient into Routine Clinical Practice. Open Journal of Nursing, 10, 308-319. https://doi.org/10.4236/ojn.2020.103021

Received: February 10, 2020

Accepted: March 27, 2020

Published: March 30, 2020

Copyright (c) 2020 by author(s) and Scientific Research Publishing Inc. This work is licensed under the Creative Commons Attribution International License (CC BY 4.0).

http://creativecommons.org/licenses/by/4.0/

\begin{abstract}
Aims: Research the process of organizational change to understand how interventions such as appearance care can be integrated into daily practice. Background: Typical treatment of breast cancer is surgery, often followed by chemotherapy. Associated aesthetic ramifications, including hair loss, frequently cause patient distress. Methods: We conducted face-to-face semi-structured hour-long recorded interviews with three nurses. We then analyzed the developmental process according to the core competencies for interprofessional collaborative practice using the thematic content analysis. Results: Nurses worked with interdisciplinary team members with mutual respect and shared values such as concern for patients' quality of life. Nurses used knowledge of appearance-related side effects combined with beautician-provided coping skills. Intervention involved responsible communication with other health professionals and patients in a team approach. Nurses promoted the developmental process and became program facilitators. Conclusion: This group-intervention program, facilitated by nurses, was integrated into daily practice. Implications for nursing management: Research partnerships between academics, clinical nurses, nurse managers, and beauticians can improve the integration of interventions in routine practice and increase awareness of patients' needs.
\end{abstract}

\section{Keywords}

Breast Cancer, Chemotherapy, Group Intervention, Interprofessional 


\section{Introduction}

The survival rate of patients with cancer increased due to early detection and improved treatment; this was made possible by medical advancement [1]. Breast cancer is the most frequently diagnosed cancer among women, with an estimated 1.7 million cases worldwide in 2012 [2]. An estimated 252,710 new cases of invasive breast cancer were diagnosed in America in 2017 [3]. In Japan, the highest incidence cancer in females is breast cancer [4]. Recently, breast cancer is being diagnosed early, and the typical treatment of breast cancer is surgery, often followed by chemotherapy to reduce the risk of recurrence. The chemotherapeutic regimen, if based on anthracyclines and taxanes, causes complete hair loss in most patients [5]. Associated appearance ramifications, include hair loss, eyelash loss, and facial discoloration which lead to patient distress [6] [7].

Zannini et al. (2012) found that, "Despite advances in the treatment of many side effects associated with chemotherapy, alopecia remains difficult to resolve [8]". Miyashita et al. (2014) reported that, "Medical professionals have recognized quality of life as a primary outcome in cancer patients [9]". There is a need to support appearance-related side effects to help maintain patients' daily life and social interaction. Appearance care is one component of cancer survivorship care, and it is important that care providers work together to meet the needs of their cancer patients, as their quality of life issues goes beyond medical needs to psychological, social, and spiritual needs. An interprofessional and collaborative approach is necessary to ensure that cancer patients' needs are met.

\subsection{Competencies of Interprofessional Collaborative Practice}

Interprofessional collaboration is "the process of developing and maintaining effective interprofessional working relationships with learners, practitioners, patients/clients/families, and communities to enable optimal health outcomes [10]". Elements of collaboration include respect, trust, shared decision making, and partnership. There are four competencies that comprise interprofessional collaborative practice: "1) Values/Ethics for Interprofessional Practice: work with individuals of other professions to maintain a climate of mutual respect and shared values; 2) Roles/Responsibilities: use knowledge of one's own role and those of other professions to appropriately assess and address the healthcare needs of the patients and populations served; 3) Interprofessional Communication: communicate with patients, families, communities, and other health professionals in a responsive and responsible manner that supports a team approach to the maintenance of health and the treatment of disease; 4) Teams and Teamwork: apply relationship-building values and the principles of team dynamics to perform effectively in different team roles to plan and deliver patient-centered care that is safe, timely, efficient, effective, and equitable [11]". 


\subsection{Translating Research into Practice}

Riley et al. (2013) stated that "What has been learned in the research setting is often not implemented into daily practice. A major challenge for practitioners is that most evidence-based interventions are not ready for widespread dissemination [12]". It may take as long as one or two decades for original research to be put into routine clinical practice. Many reasons have been offered for this slow and incomplete translation, such as research methods being complicated, and reporting standards that are not relevant to the clinical settings [13]. The appearance care program developed and evaluated by our intervention study, which was conducted for one and a half years, was chosen to be implemented into daily clinical practice when the research was terminated. The aims of this study are to describe and discuss the process of changes in the organization through research activities to understand how new interventions in research settings can become integrated into daily practice, from the perspective of nurses' proactive engagement.

\section{Methods}

\subsection{Setting}

This study was conducted in one hospital (hereafter as hospital A), which holds 520 beds and provides advanced medical treatment for outpatients and inpatients. The hospital was accredited by the Joint Commission International in 2012, and accreditation has been renewed ever since then. The development and implementation took place in the breast center and oncology center.

\subsection{Context of the Study}

To respond to the needs of breast cancer patient who were suffering from appearance-related distress, we created a team of oncology nurses, clinical psychologists, and licensed beauticians to develop an intervention program that addressed patients' health care needs along with their aesthetic care needs. The program was developed through a literature review and discussions through interprofessional collaborative practice. Through this process, the appearance care program was developed and pilot tests confirmed that the objectives of the program were met. The nurses used their knowledge of appearance-related side effects, combined with coping skills provided by the beauticians, and the principles of makeup to help maintain healthy looks during treatment.

\subsection{Study Design and Participants}

To describe the process of translating the research into practice, we conducted an exploratory case study. We approached key informants, that is, nurses who had been involved in most of the total process starting from the planning of the research design, due to the exploratory characteristic of the study. A number of patients who participated in the intervention program were 72 in total. They were also our informants, although we considered nurses as our primary partic- 
ipants for this study. Three nurses were interviewed who were involved from the planning of the research design. One was an assistant manager of the oncology center, one was a research nurse, and the other was an accredited certified nurse specialist in cancer care.

\subsection{Data Collection and Analysis}

Mari Ikela was the primary investigator (PI) of the intervention evaluation research. PI conducted face-to-face semi-structured interviews. The interview guide included four sections: patients' needs regarding appearance-related care and by whom should it be done if necessary; the effect of participating in the study; the effect of working together with non-medical professionals such as beauticians for patient care; and the effect of interacting with patients outside the outpatient clinic and what they learned from patient feedback. The interviews lasted approximately one hour for all participants and were audio recorded with participants' consent. Interview was conducted in a private room after nurses have finished their shifts. Interviews were transcribed verbatim. We also supplemented the data with conference minutes and verbatim transcripts of the expert panel meeting.

"Each transcribed interview was initially read thoroughly and notes were taken to bring out the character of the text", in accordance with the thematic content analysis procedure [14]. We used the competencies of interprofessional collaborative practice for the analysis. All significant content was extracted from the data. To secure the validity of the analysis, the co-authors were involved throughout the entire process to achieve consensus at the word, sentence, and discourse levels.

\subsection{Ethical Considerations}

The study protocol was reviewed and approved by the IRB of the author's institution. The approval was received in accordance with the ethical statements provided by IRB in accordance with the Declaration of Helsinki.

\section{Results}

\subsection{The Study Overview}

The appearance care program (here after referred as ACP) consisted of a group intervention facilitated by the nurse, aiming to build values and apply the principles of team dynamics to collaborate effectively and provide patient-centered care that is safe, timely, and effective. In studies reported elsewhere, we have designed and initiated the ACP and evaluated it carefully. After completion of the research with a positive outcome, the clinical setting chose to continue the program as an aspect of daily clinical practice.

\subsection{The Flow of Stages to "Translating Research into Practice."}

Figure 1 presents the flow of stages to "Translating Research into Practice." 


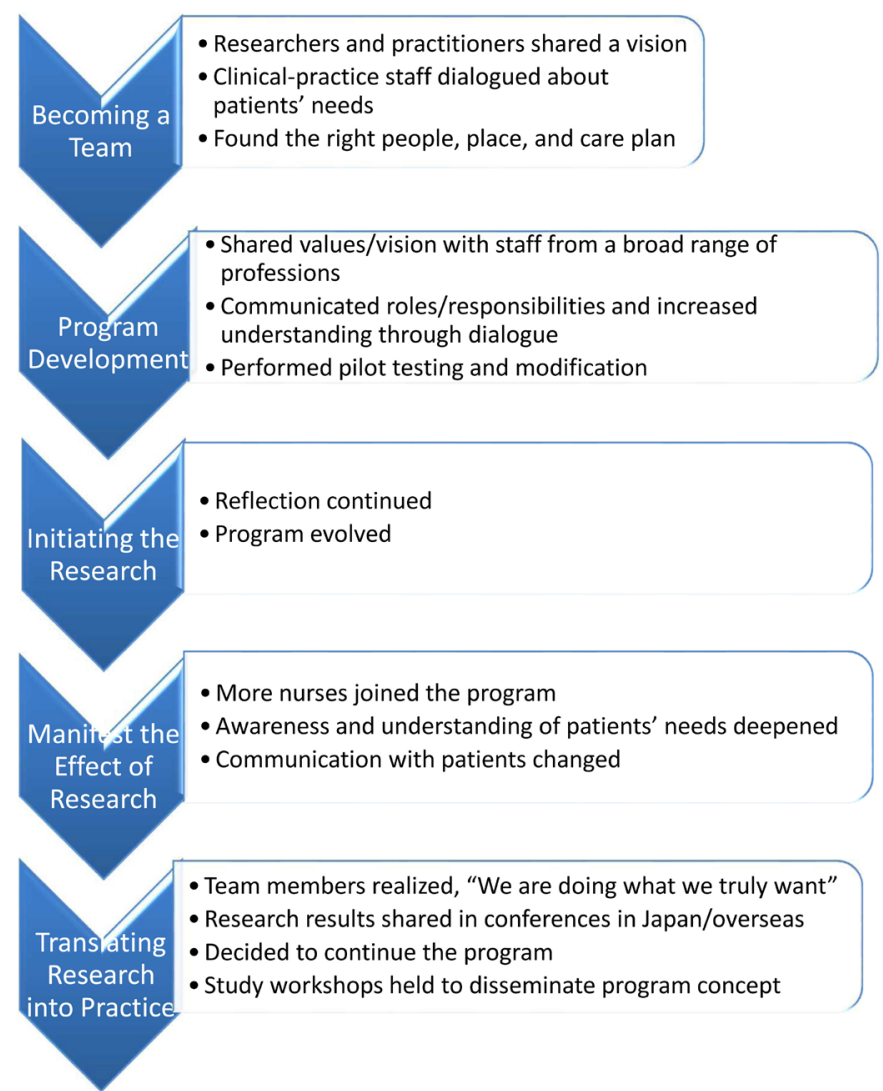

Figure 1. Flow of stages to "translating research into practice".

Before forming the expert panel, we conducted a literature review on appearance care for patients undergoing chemotherapy. Theories of learning and coping were reviewed to decide upon the model of care intervention.

\subsubsection{Becoming a Team}

\section{Researchers and practitioners shared a vision}

We met with certified beauticians and a cancer survivor who were conducting salons separately once a month on a volunteer basis at the hospital A. The survivor was a cosmetics journalist who gave make-over lessons. The attendance of patients for the salons was low and sometimes no patients came. However, the feedback from the attendees was always very positive. The volunteers were looking for other ways to disseminate their techniques to patients. PI arranged an appointment with an oncologist and the nurse manager of the breast center to discuss the possibility of designing a study to evaluate a program that focused on appearance care by an interprofessional team including nursing staff members. After thorough discussions, the hospital officials decided to allow the research to take place.

\section{What patients want}

Patients who go through chemotherapy lose not only hair from their heads but also from their eyelashes and eyebrows, which alters their facial appearance. Nurses made comments such as, "I have seen so many of them (patients) suffer 
from the change in appearance. I wished I had some approach to ease their suffering but didn't have any. I felt useless in regard to this matter" and "Teaching them how to apply make-up... Just that, is not the nurses' job, I guess." Nurses working closely with patients at the breast center had been wanting to do something about these side effects.

Finding the right person, place, and care content

The research team decided to have nurses involved in the appearance care program so it would receive more attention from patients. The researcher and nursing managers discussed the size of the group for the program and its frequency, which led to the research planning stage.

\subsubsection{Program Development}

\section{Sharing values/ vision with a broad range of professionals}

In the kick-off meeting, team members recognized that although our approaches to achieving the goal, that is, to developing a meaningful program for the patients, seemed different, we shared the same ultimate goal.

Communicate one's role and responsibilities and understand each other through "dialogue"

The team placed importance on "dialogue" instead of "discussion" to search for the content that patients needed. Beauticians emphasized shortening the period of psychological uneasiness wherein patients felt less like themselves because of their altered appearance and providing ideas for new hairstyles to lift their depressed spirits. Nursing staff, on the other hand, were astonished by the beauticians' ideas, and emphasized the safety and coexistence of nursing care.

Dialogue went on vigorously among the members of the research team, and through these processes, members understood other professionals' points of view and maintained an atmosphere of mutual respect and shared values. The team held a total of eleven meetings to develop the pilot version of the program.

Pilot testing and modification

One course of the program consisted of three sessions. Each session used a booklet including a worksheet for self-recording reflections, information on skincare, hair styling, and make-up. The program was facilitated by a nurse with the help of beauticians and a cancer survivor. Sessions started with sharing their current thoughts, followed by short lectures from a nurse and a beautician, then finished with group discussion. We conducted pilot test research sessions and thoroughly reviewed the feedback from participants and made necessary modifications, such as to session lengths (two or three hours), frequency (two or three session every week), and content.

\subsubsection{Initiating the Research}

Continuous reflections

The team members of the appearance care program (ACP) were not all working at the same site. The team created a platform to share the same information among team members between the sessions, which would have been not neces- 
sary if they were all working at the same facility. The team scheduled a debriefing after each session and made sure that the information and findings from the session were carried over to the next session. Additionally, ad hoc listservs and direct telecommunications occurred during the sessions. The main facilitator of the program remained throughout the three sessions. The team scheduled a face-to-face conference every three months with all members.

\subsubsection{Manifesting the Effects of the Research \\ Expanding team}

Two nurses, who were working at the research setting, were on the research team at the beginning. They were trained to provide the program. As the research continued, nurses who noticed changes in the patients at the breast center requested to become part of the provider team. The changes nurses observed in patients were that they were more proactive toward treatment and seemed relaxed with their changing appearance because they had acquired new skills. By the end of the research period, the nurses involved had tripled in number.

\section{Advancement of knowledge of patients' needs}

Nurses who participated in providing the program were surprised by how many difficulties patients had from issues of appearance. When patients visited an ambulatory chemotherapy station, nurses usually asked questions about their health condition. Appearance issues seldom came up as a topic. Those who were involved in the ACP with patients realized that appearance issues are critical because they are part of daily life outside the hospital. The nurses modified their questions so that the patients were able to consult about a broad range of difficulties they were facing. Knowing the patients through the program also made the nurses feel comfortable talking in outpatient care settings.

\subsubsection{Translating Research into Practice}

\section{An epochal event}

The research was designed to conduct the program in a group, with a minimum of three persons, because we developed the program to promote a peer-support effect. MI received a call from the hospital about whether they could provide the program even if there was only one participant. PI said, "Why not?" and conducted the program anyway. This event made the team realize that they were practicing something that they had truly been wanting to do. The team shared the same goal, and if anyone wanted to participate, they were welcome.

\section{Decision to continue the program}

The research team presented the effects of the program in various conferences, both domestically and overseas. The program received public response and many providers in medical fields came to observe; members of the research team were requested to write articles about it. The research team received positive feedback from patients, who reported that the program helped them become ready for bodily changes, provided useful information, enabled interaction with family members, and provided effective peer support. 
We decided to incorporate these research activities into regular care. In-house workshops for other nurses were held to promote and disseminate the program.

\section{Discussion}

This group intervention program was carried out in the hospital with professionals from different backgrounds and cancer survivors as well. Most previous interventions were performed by nurses or beauticians alone. The process of developing the program to its actual implementation is described according to the competencies claimed by Association of American Medical Colleagues: "1) Values/Ethics for Interprofessional Practice, 2) Roles/Responsibilities, 3) Interprofessional Communication, and 4) Teams and Teamwork."

\subsection{Value/Ethics for Interprofessional Practice}

Since the late 1990s, chemotherapeutic regimens based on anthracyclines and taxanes have been introduced. This therapy causes complete alopecia in most patients. Many women consider alopecia to be one of the most painful and scared side effects of chemotherapy. Nurses looking for ideas to ease patients' pain attended classes to learn how to hide chemotherapy changes with make-up, which was called a "rehabilitation make-over." However, this was difficult to implement in daily care. The nurses were interested in what volunteers were providing but had never shared their thoughts. The research introduction was a turning point for both nurses and volunteers/survivors. It is rare for interprofessionals such as nurses, doctors, beauticians, and survivors to form a team together and develop an intervention program. The current research proposal was a good match for the nurses' goals, and the content of the intervention was discussed between different professionals, leading to increased competency.

\subsection{Roles/Responsibilities}

When women learn that they would lose their hair during chemotherapy, they start anticipating the impact of hair loss on themselves and people around them. Anticipation of an undesired event can cause anxiety, fear, or even depression. This is more so if women feel there are no other options resolve this issue. Anticipatory coping is the process of anticipation with preparation for an event. By taking specific actions to determine how they will look without hair, how they would manage their appearance in social environment, women gain a greater sense of control over their changing appearance. Borsellino et al. (2011) reported, "This greater sense of control may ease or lessen feelings of fear and depression and help women to take control of other aspects of their lives that contribute to a greater quality of life during cancer treatment [15]". The core concept of the program thus focused on proactive coping skills [16] and integrated the anticipatory coping skills to promoting peer support through the process of self-reflection. It is important to provide timely and accurate information about the side effects of treatment and the potential for anticipatory coping strategies 
to reduce distress [17]. Group intervention provides women with the opportunity to learn with other women in a similar situation. "This may help to alleviate the sense of loneliness and provide additional social support [18]".

Borsellino et al. (2011) discussed that, "Oncology nurses are in a unique position to help women turn their anxiety about hair loss into an anticipatory coping process, one that increases women's sense of control over some of the outward changes taking place in their bodies [15]". This may lead women to be more proactive toward the treatment of cancer.

\subsection{Interprofessional Communication}

The research team set regular meetings at the beginning of the new cycle of sessions. The meetings were purposefully planned; otherwise there would be no chance to exchange information. These interactions were done to confirm the goals and vision of the ACP. Thus, practical knowledge was accumulated through interprofessional discussions.

\subsection{Teams and Teamwork}

The nurse manager had control over staff shifts, so three other nurses were able to join as providers of the program. The nurse manager utilized her position of power to expand the providing team. The interaction between nurses and beauticians created wider perspectives on the possibilities for providing care to patients. The session led by a nurse contained information introduced at the time of orientation to chemotherapy, although orientation was mainly done orally and while the patients were in a stage of denial, a common defense mechanism. By developing the ACP, awareness was built while interacting with peers on the same path. The process of anticipation became visual and was explained interactively in the program.

A limitation of this study is that the study is one case study with the hospital A and for the feasibility, other observation and requirements may have to be considered.

\section{Conclusion}

This interprofessional collaborative practice designed for breast cancer patients focused on appearance care, which is provided in a group intervention facilitated by a nurse, beauticians, and the survivor. The research activities to provide new interventions were integrated into daily practice from the perspective of nurses' proactive engagement.

\section{Implication for Nursing Management}

Even if it turns out that a new approach developed in nursing research produces good results, it is often not continued in daily care. In this study, however, the researcher joined forces with a nurse who felt the need for new form of everyday care at the clinical site and a beauty specialist who wanted to support cancer pa- 
tients cosmetically. The program started with the sharing of a vision of what support nurses wanted to provide for the patients. Nurses asked about how to approach patients, re-examined their usual care methods, and created a new approach through dialogue. The nurses and beauticians were able to recognize that they were a team looking out for patients' well-being, even though team members came from different disciplines and had different points of view. The participating nurses realized that their care deepened their understanding of patients and promoted communication, and nurses who did not participate also noticed the changes in patients who received care. Nurse managers encouraged nurses to be involved in the provision of the program, and through a series of processes, they developed what began as research into everyday care. This research highlights the value of nursing research partnerships between academics, clinical nurses, nurse managers, and beauticians, to improve the integration of new interventions into routine practice and increase awareness of the needs of patients.

\section{Acknowledgements}

We would like to thank all the participants and medical staff who participated in this study. Special thanks to Ms. Takako Yamazaki, Mr. Shuhei Ueno and Ms. Ichiyo Otani for providing beauty care in the program. We are also grateful to Ms. Kazuko Kondo for her role as the first coordinator of appearance care in a hospital. This study was funded by the St. Luke's Life Science Institute, Tokyo and JSPS KAKENHI Grant Number JP18H03069. The funding agency was not involved in the design and conduct of the study, nor in the collection, management, analysis, interpretation of the data, and not involved in the writing of the manuscript.

\section{Conflicts of Interest}

The authors declare no conflicts of interest associated with this manuscript.

\section{Ethical Approval}

The study protocol and the assessment procedures were reviewed and approved by the Ethics Committee of the University of Tokyo (\#10452) and St. Luke International Hospital (14-R006).

\section{References}

[1] Youlden, D.R., Cramb, S.M., Dunn, N.A., Muller, M.J., Pyke, C.M. and Baade, P.D. (2012) The Descriptive Epidemiology of Female Breast Cancer: An International Comparison of Screening, Incidence, Survival and Mortality. Cancer Epidemiology, 36, 237-248. https://doi.org/10.1016/j.canep.2012.02.007

[2] Torre, L.A., Bray, F., Siegel, R.L., Ferlay, J., Lortet-Tieulent, J. and Jemal, A. (2015) Global Cancer Statistics, 2012. CA: A Cancer Journal for Clinicians, 65, 87-108. https://doi.org/10.3322/caac.21262

[3] American Cancer Society (2017) Breast Cancer Facts and Figures 2017-2018. 
American Cancer Society, Inc., Atlanta.

https://www.cancer.org/content/dam/cancer-org/research/cancer-facts-and-statistic s/breast-cancer-facts-and-figures/breast-cancer-facts-and-figures-2017-2018.pdf

[4] Katanoda, K., Matsuda, T., Matsuda, A., et al. (2013) An Updated Report on the Trends in Cancer Incidence and Mortality in Japan. Japanese Journal of Clinical Oncology, 43, 492-507. https://doi.org/10.1093/jjco/hyt038

[5] Rosati, M.S., Raimondi, C., Baciarello, G., Grassi, P., Giovannoni, S., Petrelli, E., Basile, M.L., Girolami, M., Di Seri, M. and Frati, L. (2011) Weekly Combination of Non-Pegylated Liposomal Doxorubicin and Taxane in First-Line Breast Cancer: wALT Trial (Phase I-II). Annals of Oncology, 22, 315-320. https://doi.org/10.1093/annonc/mdq392

[6] Fall-Dickson, J.M. and Rose, L. (1999) Caring for Patients Who Experience Chemotherapy-Induced Side Effects: The Meaning for Oncology Nurses. Oncology Nursing Forum, 26, 901-907.

[7] Carelle, N., Piotto, E., Bellanger, A., Germanaud, J., Thuillier, A. and Khayat, D. (2002) Changing Patient Perceptions of the Side Effects of Cancer Chemotherapy. Cancer, 95, 155-163. https://doi.org/10.1002/cncr.10630

[8] Zannini, L., Verderame, F., Cucchiara, G., Zinna, B., Alba, A. and Ferrara, M. (2012) “My Wig Has Been My Journey's Companion": Perceived Effects of an Aesthetic Care Programme for Italian Women Suffering from Chemotherapy-Induced Alopecia. European Journal of Cancer Care, 21, 650-660. https://doi.org/10.1111/j.1365-2354.2012.01337.x

[9] Miyashita, M., Ohno, J., Kataoka, A., Tokunaga, E., Masuda, N., Shien, T., Kawabata, K. and Takahashi, M. (2015) Unmet Information Needs and Quality of Life in Young Breast Cancer Survivors in Japan. Cancer Nursing, 38, E1-E11. https://doi.org/10.1097/NCC.0000000000000201

[10] Health Canada (2010) A National Interprofessional Competency Framework. Canadian Interprofessional Health Collaborative.

https://phabc.org/public-health-core-competency-development/resources/cihc-nati onal-interprofessional-competency-framework/

[11] Interprofessional Education Collaborative Expert Panel (2011) Core Competencies for Interprofessional Collaborative Practice. Report of an Expert Panel, Washington DC.

https://www.aacom.org/docs/default-source/insideome/ccrpt05-10-11.pdf?sfvrsn=7 7937f97_2

[12] Riley, W.T., Glasgow, R.E., Etheredge, L. and Abernethy, A.P. (2013) Rapid, Responsive, Relevant (R3) Research: A Call for a Rapid Learning Health Research Enterprise. Clinical and Translational Medicine, 2, 10.

https://doi.org/10.1186/2001-1326-2-10

[13] Neta, G., Glasgow, R.E., Carpenter, C.R., Grimshaw, J.M., Rabin, B.A., Fernandez, M.E. and Brownson, R.C. (2015) A Framework for Enhancing the Value of Research for Dissemination and Implementation. American Journal of Public Health, 105, 49-57. https://doi.org/10.2105/AJPH.2014.302206

[14] Braun, V. and Clarke, V. (2006) Using Thematic Analysis in Psychology. Qualitative Research in Psychology, 3, 77-101. https://doi.org/10.1191/1478088706qp063oa

[15] Borsellino, M. and Young, M.M. (2011) Anticipatory Coping. Clinical Journal of Oncology Nursing, 15, 311-315. https://doi.org/10.1188/11.CJON.311-315

[16] Aspinwall, L.G. and Taylor, S.E. (1997) A Stitch in Time: Self-Regulation and Proactive Coping. Psychology Bulletin, 121, 417-436. 
https://doi.org/10.1037/0033-2909.121.3.417

[17] Gibbons, A. and Groarke, A. (2018) Coping with Chemotherapy for Breast Cancer: Asking Women What Works. European Journal of Oncology Nursing, 35, 85-91. https://doi.org/10.1016/j.ejon.2018.06.003

[18] Jones, J.M., Cheng, T., Jackman, M., Walton, T., Haines, S., Rodin, G. and Catton, P. (2013) Getting Back on Track: Evaluation of a Brief Group Psychoeducation Intervention for Women Completing Primary Treatment for Breast Cancer. Psychooncology, 22, 117-124. https://doi.org/10.1002/pon.2060 